\title{
MARKOV OPERATORS, PEAK POINTS, AND CHOQUET POINTS
}

\author{
ROBERT E. ATALLA
}

\begin{abstract}
We deal with conditions on a closed subspace $\boldsymbol{L}$ of $C(X)$ under which weak peak points are equivalent with Choquet points. If $\boldsymbol{L}$ satisfies a strengthened form of this equivalence ('weak peak sets $=$ Choquet sets'-definitions are given below), then for any image of $\boldsymbol{L}$ under a Markov projection, weak peak points and Choquet points are equivalent. Conditions under which $\boldsymbol{L}$ satisfies the strengthened equivalence include (i) $\boldsymbol{L}$ interpolates its Shilov boundary, and (ii) $\boldsymbol{L}$ is the space of fixed points of a Markov operator $T$ on $C(X)$, where $T$ is in an appropriate sense concentrated on the Shilov boundary of $\boldsymbol{L}$.
\end{abstract}

1. Introduction. Let $C(X)$ be the space of real continuous functions on the compact $T_{2}$ space $X$, and let $T$ be a Markov operator on $C(X)$, i.e., a nonnegative continuous operator with $T e=e$ ( $e$ the unit function). For each $x \in X$, let $t_{x}$ be the Borel probability measure such that $T f(x)=$ $\int f d t_{x}(f \in C(X))$. Let $\boldsymbol{F}_{T}=\{f \in C(X): T f=f\}$. If $x \in X$ and $f \in \boldsymbol{F}_{T}$, then $f(x)=\int f d t_{x}$ so that, in the language of 'function algebras', the measure $t_{x}$ 'represents' $x$ with respect to the linear space $\boldsymbol{F}_{T}$. Apparently the relevance of such ideas to the theory of Markov operators was first noted by S. P. Lloyd [LI], and extensively developed by R. Sine [S, §4].

In this paper we show that under suitable hypotheses the real linear space $\boldsymbol{F}_{T}$ has an interesting property in common with complex function algebras. Bishop showed that for such algebras, the Choquet points of $X$ coincide with the weak peak points. (See, e.g., [L, p. 165, Exercise 1]. A weak peak point is the intersection of peak sets.) For linear subspaces of $C(X)$, the best that can be said in general is that (at least if $X$ is metrizable) peak points are dense in the Choquet points [P, pp. 54-57]. We show that if $L_{1}$ is a closed subspace of $C(X)$ which satisfies a strengthened form of Bishop's equivalence, and $\boldsymbol{L}_{2} \subset \boldsymbol{L}_{1}$ is the range of a Markov projection $P$ on $L_{1}$ (i.e., $L_{2}=F_{P}$ ), then $L_{2}$ satisfies Bishop's equivalence.

Presented in part to the Society, April 18, 1973 under the title Characterizations of peak sets; received by the editors November 27, 1972 and, in revised form, February 26, 1973.

AMS (MOS) subject classifications (1970). Primary 46E15, 46J10, 47B55.

Key words and phrases. Markov operator, projection, $C(X)$, Shilov boundary, peak point, Choquet point, representing measure, fixed points of a Markov operator.

(c) American Mathematical Society 1973 
The rest of the paper is devoted to conditions under which a linear space satisfies the strengthened equivalence ('weak peak sets coincide with Choquet sets'-definitions are given in 2.1 , below). We show that if $\boldsymbol{L}$ is a closed linear subspace of $C(X)$ containing the constants, and if $\boldsymbol{L} \mid S=C(S)$, where $S$ is the Shilov boundary for $L$, then $L$ satisfies the strengthened equivalence. Examples of such $\boldsymbol{L}$ include $C(X)$ itself, and the space of functions continuous on the closed unit disc and harmonic on the open unit disc. In the final section we show that if $T$ is a Markov operator on $C(X)$, and certain hypotheses are satisfied, then the above results apply to $\boldsymbol{F}_{T}$. Hence in this case every image of $\boldsymbol{F}_{T}$ under a Markov projection satisfies Bishop's equivalence.

\section{Peak sets and Choquet sets.}

2.1 Definitions. Let $\boldsymbol{L}$ be a linear subspace of $C(X)$. A closed set $F$ is called a peak set if there exists $f \in \boldsymbol{L}$ with $f=1$ on $F$ and $|f|<1$ off $F$. $F$ is a weak peak set if it is the intersection of peak sets.

If $x \in X$, a Borel probability measure $m$ on $X$ is said to represent $x$ if $f(x)=\int f d m$ for all $f \in \boldsymbol{L}$. The set of representing measures for $x$ is denoted by $H_{x}=H_{x}(L)$.

A closed set $F$ is called a Choquet set if (i) $x \in F$ and $m \in H_{x}$ imply $\operatorname{car}(m) \subset F$ (where $\operatorname{car}(m)$ is the smallest closed set of measure 1), and (ii) $x \notin F$ and $m \in H_{x}$ imply $\operatorname{car}(m) \not F$. (Condition (i) was suggested by the definition of a self-supporting set in the theory of Markov operators- $F$ is self-supporting if $x \in F$ implies $\operatorname{car}\left(t_{x}\right) \subset F$. The connection between Choquet sets and weak peak sets was suggested by Sine's observation [S, Theorem 1.2] that a peak set for $\boldsymbol{F}_{T}$ is self-supporting.)

$x \in X$ is a Choquet point if the point mass $\delta_{x}$ at $x$ is an extreme point of the set $\left\{\rho \in L^{\prime}: \rho(e)=\|\rho\|=1\right\}$, or, equivalently, $H_{x}=\left\{\delta_{x}\right\}[\mathbf{L}, \mathbf{p} .52]$. Note that $x$ is a Choquet point iff $\{x\}$ is a Choquet set.

If $\boldsymbol{L}$ is a linear subspace of $C(X)$ which fails to separate points, let $D$ be the set of equivalence classes in $X$ induced by $L$. That is, if $x \in D \in D$, then $D=\{y: f(y)=f(x)(f \in \boldsymbol{L})\}$. In a natural way, we may think of $\boldsymbol{L}$ as a space of functions on $D$, and hence may think of each $D \in D$ as a point mass $\delta_{D} \in \boldsymbol{L}^{\prime}\left(\delta_{D}(f)=f(D)\right)$. As before, $D$ is a Choquet point if it is an extreme in $\left\{\rho \in L^{\prime}: \rho(e)=\|\rho\|=1\right\}$.

2.2 Lemma. If $L$ is a linear subspace of $C(X)$, then every weak peak set for $L$ is a Choquet set.

Proof. First, let $F$ be a peak set, say $F=f^{-1}(1)$ with $|f|<1$ off $F$. Let $x \in X$ and $m \in H_{x}$. Then (cf. [S, Theorem 2.1]) $1=f(x)=\int f d m$. Since $f \leqq 1$, we have $f=1$ on $\operatorname{car}(m)$, so $\operatorname{car}(m) \subset F$. If $x \notin F$ and $m \in H_{x}$, suppose $\operatorname{car}(m) \subset F$. Then $f(x)=\int f d m=1$, which is impossible. Hence $\operatorname{car}(m) \not f$, 
and $F$ is proved to be a Choquet set. Now if we show that the intersection of Choquet sets is again a Choquet set, it will follow that every weak peak set is a Choquet set. Let $F=\bigcap\left\{F_{a}: a \in A\right\}$, where each $F_{a}$ is a Choquet set. If $x \in F$ and $m \in H_{x}$, then $x \in F_{a}$ for each $a \in A$, and hence $\operatorname{car}(m) \subset F_{a}$ for each $a \in A$. Hence $\operatorname{car}(m) \subset F$. If $x \notin F$ and $m \in H_{x}$, then $x \notin F_{a}$ for some $a \in A$. Since $F_{a}$ is Choquet, $\operatorname{car}(m) \not \subset F_{a}$, and hence $\operatorname{car}(m) \not \subset F$.

2.3 THEOREM. Let $L_{1}$ be a closed subspace of $C(X)$ which contains the constants, and assume that the weak peak sets for $L_{1}$ coincide with the Choquet sets. Suppose $L_{2} \subset L_{1}$ is the range of a Markov projection $P$ on $L_{1}$, and let $D$ be the space of equivalence classes under $L_{2}$. If $D \in D$, the following are equivalent

(a) $D$ is a Choquet point for $\boldsymbol{L}_{2}$,

(b) $D$ is a weak peak set for $L_{2}$,

(c) $x \in D$ and $m \in H_{x}=H_{x}\left(L_{2}\right)$ imply $\operatorname{car}(m) \subset D$.

Proof. The equivalence of (a) and (c) is known [L, Theorem 9, p. 51]. (In fact, it holds for any closed linear subspace of $C(X)$.)

(b) implies (c). This is part of Lemma 2.2.

(c) implies (b). Let $D$ satisfy condition (c), and let $E \in D$ be distinct from $D$. We shall show that there is a peak set $F$ (for $L_{2}$ ) with $D \subset F$ and $E \cap F=\varnothing$.

First we show that $D$ is a Choquet set for $\boldsymbol{L}_{2}$. By hypothesis, condition (i) for a Choquet set is satisfied. For condition (ii), let $x \notin D$. Then $x \in F$ for some $F \in D$. If $m \in H_{x}$ and $\operatorname{car}(m) \subset D$, then $f(F)=f(x)=f(D)$ for all $f \in \boldsymbol{L}_{2}$, which is impossible. Hence $\operatorname{car}(m) \not \subset D$.

Since $L_{2} \subset L_{1}$, any representing measure for a point with respect to $L_{1}$ is also a representing measure with respect to $L_{2}$. Hence $D$ is a Choquet set for $\boldsymbol{L}_{1}$ as well as for $\boldsymbol{L}_{2}$. By hypothesis, $D$ is a weak peak set for $\boldsymbol{L}_{1}$. Let $x \in E$ and $p_{x}$ be any probability measure on $X$ representing the functional $f \rightarrow P f(x)\left(f \in \boldsymbol{L}_{1}\right)$. Since $P f=f$ for $f \in \boldsymbol{L}_{2}, p_{x} \in H_{x}\left(\boldsymbol{L}_{2}\right)$. Since $x \notin D$ and we have shown $D$ to be a Choquet set for $L_{2}$, we have $\operatorname{car}\left(p_{x}\right) \notin D$. Choose $y \in \operatorname{car}\left(p_{x}\right) \backslash D$. Since $D$ is a weak peak set for $L_{1}$, there exists $f \in L_{1}$ such that $D \subset F=f^{-1}(1), f \leqq 1$, and $f(y)<1$. Then $P f \in L_{2}, P f \leqq 1$, and $P f(E)=P f(x)=\int f d p_{x}<1$. Moreover if $z \in D$ and $p_{z}$ represents $f \rightarrow P f(z)\left(f \in L_{1}\right)$, then we have $\operatorname{car}\left(P_{z}\right) \subset D \subset f^{-1}(1)$, whence $P f(D)=P f(z)=1$. It follows that $D$ is a weak peak point for $L_{2}$.

2.4 ExAmples. (a) $C(X)$ itself satisfies our conditions on $L_{1}$, so that every image of $C(X)$ under a Markov projection satisfies Bishop's equivalence. Actually, a stronger result is available in this case (Corollary 3.5). (b) Let $\boldsymbol{L}$ be the real functions which are continuous on the closed unit disc and harmonic on the open unit disc. All weak peak sets are peak sets, and the class of peak sets is exactly the class of closed proper subsets of 
the unit circle. This is also the class of Choquet sets. (Note that if $|z|=1$, then the only element of $H_{z}$ is the point mass at $z$, and if $|z|<1$, then the Poisson integral gives a representing measure carried by the whole unit circle, but not any proper subset thereof.) Hence any image of $\boldsymbol{L}$ under a Markov projection satisfies Bishop's equivalence.

This example suggests the following result.

2.5 THEOREM. Let $\boldsymbol{L}$ be a closed linear subspace of $C(X)$ which separates points and contains the constants. If $L \mid S=C(S)$, where $S$ is the Shilov boundary for $\boldsymbol{L}$, then the class of weak peak sets for $\boldsymbol{L}$ coincides with the class of Choquet sets.

Proof. By Lemma 2.2, weak peak sets are Choquet sets. For the reverse inclusion, let $F$ be a Choquet set, and let $x \notin F$. We must show that there is a peak set $G$ with $F \subset G$ and $x \notin G$. Since each $y \in X$ has a representing measure with support in $S$, and $F$ is a Choquet set, it follows that $F \cap S$ is a nonvoid closed subset of $S$. Choose $m \in H_{x}$ such that $\operatorname{car}(m) \subset S$. Since $x \notin F$ and $F$ is a Choquet set, $\operatorname{car}(m) \not F \cap S$. Choose $y \in \operatorname{car}(m) \backslash F \cap S$, and choose $f \in C(S)$ such that $f=1$ on $F \cap S, f(y)=0$, and $0 \leqq f \leqq 1$. Let $g \in \boldsymbol{L}$ be such that $g \mid S=f$. Now (i) $0 \leqq g \leqq 1$, (ii) $g(x)=\int g d m<1$, and (iii) if $z \in F$, then there exists $n \in H_{z}$ such that $\operatorname{car}(n) \subset F \cap S$ and (since $f=1$ on $F \cap S) g(z)=\int g d n=\int f d n=1$. Thus if $G=g^{-1}(1)$, then $F \subset G$ and $x \notin G$.

2.6 Remark. We note that for function algebras, weak peak sets and Choquet sets need not coincide. For instance if $\boldsymbol{A}$ is the algebra of functions continuous on the closed unit disc and analytic on the open disc, then the representing measures for $\boldsymbol{A}$ are the same as those for the harmonic functions (Example 2.4(b)), so that Choquet sets are again the proper closed subsets of the unit circle. However a closed arc on the unit circle cannot be a peak set for $\boldsymbol{A}$ [L, p. 64, Exercise 1]. Moreover, better results cannot be obtained by replacing peak sets by max-mod sets.

\section{The fixed points of a Markov operator.}

3.1 Remarks. In this section $T$ will be a Markov operator on $C(X)$, $\boldsymbol{F}_{T}=\{f: T f=f\}$, and $X \mid \boldsymbol{D}$ the collection of constancy sets for $\boldsymbol{F}_{T}$, endowed with the quotient topology. $\boldsymbol{F}_{T}$ induces a point separating linear space of functions $\rho\left(\boldsymbol{F}_{T}\right)$ on $X / \boldsymbol{D}$. Suppose $T$ is mean-stable, i.e., $(1 / n)\left(T+\cdots+T^{n}\right)$ $\rightarrow P$ in the strong operator topology, where $P$ is a Markov projection. Then $\boldsymbol{F}_{T}=\boldsymbol{F}_{P}$, and it follows from Theorem 4.2(a) of [S] that $\rho\left(\boldsymbol{F}_{T}\right) \mid S=$ $C(S)$, where $S$ is the Shilov boundary for $\rho\left(F_{T}\right)$ in $X / D$, and hence Choquet sets for $\rho\left(F_{T}\right)$ coincide with weak peak sets. In Theorem 3.4 and Corollary 3.5 below we show that the same results hold even if $T$ is not mean stable, if we assume that the Choquet boundary for $\rho\left(\boldsymbol{F}_{T}\right)$ is closed in $X / D$, and $T$ is in a sense concentrated on this boundary. 
3.2 Definition. $M_{T}=\{g: T(f g)=(T f)(T g)(f \in C(X))\}$. In Theorem 1.2 of $[\mathbf{A}], M_{T}$ is characterized as exactly those elements of $C(X)$ which are constant on each set $\operatorname{car}\left(t_{x}\right)(x \in X)$, where $T f(x)=\int f d t_{x}$.

3.3 LEMMA. The following are equivalent

(a) $\boldsymbol{F}_{T}$ is an algebra,

(b) $\boldsymbol{F}_{T} \subset \boldsymbol{M}_{T}$,

(c) If $x \in D \in D$, then $\operatorname{car}\left(t_{x}\right) \subset D$.

Proof. (a) implies (b). If $\boldsymbol{F}_{\boldsymbol{T}}$ is an algebra, then $T f=f$ implies $T\left(f^{2}\right)=(T f)^{2}$. By Theorem 1.3 of [A] we have $f \in M_{T}$. Hence $\boldsymbol{F}_{T}, M_{T}$.

(b) implies (a). If $f, g \in \boldsymbol{F}_{T} \subset \boldsymbol{M}_{T}$, then $T(f g)=(T f)(T g)=f g$, so $f g \in \boldsymbol{F}_{T}$, i.e., $\boldsymbol{F}_{\boldsymbol{T}}$ is an algebra.

(a) implies (c). If (c) fails, then there exists $D \in D$ and $x \in D$ with car $\left(t_{x}\right)$ not contained in $D$. Choose $E \in D$ with $E \neq D$ and $\operatorname{car}\left(t_{x}\right) \cap E$ nonvoid. If $\boldsymbol{F}_{\boldsymbol{T}}$ is an algebra, then there exists nonnegative $f \in \boldsymbol{F}_{T}$ with $f=0$ on $D$ and $f=1$ on $E$. (Note that $\boldsymbol{F}_{\boldsymbol{T}}$, contains the constants.) Hence $f(x)=0$ and $T f(x)>0$, so $T f(x) \neq f(x)$, a contradiction. So $\boldsymbol{F}_{T}$ is not an algebra, i.e., (a) fails.

(c) implies (b). If $x \in X$ and (c) holds, then $\operatorname{car}\left(t_{x}\right)$ is contained in a constancy set for $F_{T}$, and hence each $f \in F_{T}$ is constant on $\operatorname{car}\left(t_{x}\right)$. By [A, Theorem 1.2], we have $\boldsymbol{F}_{T} \subset \boldsymbol{M}_{T}$.

3.4 THEOREM. Suppose (i) the Choquet boundary for $\rho\left(\boldsymbol{F}_{T}\right)$ in $X / D$ is closed, and (ii) for each $x \in X, \operatorname{car}\left(t_{x}\right) \subset \pi^{-1}(S)$, where $\pi|X \rightarrow X| D$ is the quotient map, and $S$ is the Shilov boundary for $\rho\left(F_{T}\right)$ in $X / D$. Then $\rho\left(\boldsymbol{F}_{T}\right) \mid S=C(S)$.

Proof. Since the Choquet boundary is always dense in the Shilov boundary [L, p. 49], (i) implies that they are equal. Now $\pi^{-1}(S)=\bigcup\{D \in$ $\boldsymbol{D}: D$ a Choquet point in $X \mid \boldsymbol{D}\}$, and by continuity of $\pi, \pi^{-1}(S)$ is closed in $X$. If $x \in D \in D$, where $D$ is a Choquet point, then $\operatorname{car}(m) \subset D$ for each $m \in H_{x}$ [L, Theorem 9, p. 51]. Now $t_{x} \in H_{x}\left(\boldsymbol{F}_{\eta}\right)$, so $\operatorname{car}\left(t_{x}\right) \subset D$. Hence if $f \in C(X)$ and $f=0$ on $\pi^{-1}(S)$, then $T f=0$ on $\pi^{-1}(S)$, and $T$ induces in a natural way a linear operator $R$ on $C\left(S_{0}\right)$, where $S_{0}=\pi^{-1}(S)$.

We show that $\boldsymbol{F}_{R}=\boldsymbol{F}_{T} \mid S_{0}$. Clearly, the right side is contained in the left. For the reverse inclusion, let $f \in F_{R}$. We extend $f$ to a function $g$ on all $X$ as follows. If $x \in S_{0}$, let $g(x)=f(x)$. If $x \notin S_{0}$, then by assumption (ii) we have $\operatorname{car}\left(t_{x}\right) \subset S_{0}$, and we may define $g(x)=\int f d t_{x}$. Since $f \in F_{R}$, this integral formula holds for all $x \in X$. By weak-* continuity of the map $x \rightarrow t_{x}(x \in X), g \in C(X)$, and $g \mid S_{0}=f$. Moreover $g \in \boldsymbol{F}_{T^{\prime}}$, for since $\operatorname{car}\left(t_{x}\right) \subset S_{0}$ for all $x$, and $g \mid S_{0}=f$, we have $T g(x)=\int g d t_{x}=\int f d t_{x}=g(x)$. Thus $f \in \boldsymbol{F}_{T} \mid S_{0}$. 
Since $\boldsymbol{F}_{R}=\boldsymbol{F}_{T} \mid S_{0}$, it follows that the constancy sets for $F_{R}$ are precisely the sets $D \in \boldsymbol{D}$ such that $D$ is a Choquet point in $X / D$. By Theorem 2.3 each such $D$ satisfies the invariance condition (c) of Lemma 3.3, and it follows that $\boldsymbol{F}_{R}$, and hence $\boldsymbol{F}_{T} \mid S_{0}$, is a closed algebra containing the constants and separating the sets $\boldsymbol{D}$. By Stone-Weierstrass we get $\rho\left(\boldsymbol{F}_{T}\right) \mid S=C(S)$.

3.5 COROLlary. Under the hypotheses of 3.4, weak peak sets for $\boldsymbol{F}_{T}$ coincide with Choquet sets.

Proof. It follows from 3.4 and 2.5 that weak peak sets and Choquet sets for $\rho\left(\boldsymbol{F}_{T}\right)$ in $X / \boldsymbol{D}$ coincide. Let $F$ be a Choquet set in $X$ for $\boldsymbol{F}_{T}$. We must show that $F$ is a weak peak set. But first we prove (i) $F=\bigcup\{D \in D$ : $F \cap D \neq \varnothing\}$, and (ii) $\pi(F)$ is a Choquet set in $X \mid D$ for $\rho\left(F_{T}\right)$.

(i) Suppose $D \in D$ is such that the two sets $D \cap F$ and $D \backslash F$ are both nonvoid, say $x \in D \cap F$ and $y \in D \backslash F$. If $m \in H_{x}$ and $n \in H_{y}$, then (since $F$ is a Choquet set) we have $\operatorname{car}(m) \subset F$ and $\operatorname{car}(n) \notin F$. But $x$ and $y$ are both contained in the same level set $D$, whence $H_{x}=H_{y}$, a contradiction. Thus, (i) holds.

(ii) Suppose $D \in \pi(F)$ i.e., $D \subset F$, and $m \in H_{D}\left(\rho\left(F_{T}\right)\right)$. We must show that $\operatorname{car}(m) \subset \pi(F)$. Let $A$ be the algebra of $f \in C(X)$ which are constant on each set $D \in \boldsymbol{D}$. If $f \in \boldsymbol{A}$, define $\rho f$ on $X / \boldsymbol{D}$ by $(\rho f)(D)=f(x)$, where $x \in D$. Then $\rho f \in C(X / D)$ [Sem, Proposition 5.2.5]. Since $\boldsymbol{F}_{T} \subset \boldsymbol{A}$, $\rho(A)$ is a uniformly closed point separating subalgebra of $C(X / D)$ which contains the constants. By Stone-Weierstrass we have $\rho(A)=C(X / D)$. Define the linear functional $L$ on $\boldsymbol{A}$ by $L(f)=\int \rho f d m$. By the HahnBanach and Riesz representation theorems, there exists a measure $m_{0}$ on $X$ which represents $L$. If $x \in D$, then clearly $m_{0} \in H_{x}\left(\boldsymbol{F}_{T}\right)$. Since $D \subset F$ and $F$ is a Choquet set, we have $\operatorname{car}\left(m_{0}\right) \subset F$. Now suppose $g$ is a nonnegative element of $C(X \mid D)$, and $g=0$ on $\pi(F)$. Choose $f \in C(X)$ such that $\rho f=g$. Then $f=0$ on $F$, and $\int g d m=L(f)=\int f d m_{0}=0$. It follows that $\operatorname{car}(m) \subset \pi(F)$.

If $D \notin \pi(F)$ and $m \in H_{D}\left(\rho\left(F_{T}\right)\right)$, a similar proof yields $\operatorname{car}(m) \notin \pi(F)$. It follows that $\pi(F)$ is a Choquet set in $X / D$.

To show $F$ is a weak peak set, let $x \notin F$. Then $x \in D$ for some $D \in D$ which by (i) is disjoint from $F$, i.e., $D \notin \pi(F)$. Since $\pi(F)$ is a Choquet set, and hence a weak peak set, there exists $g \in \rho\left(F_{T}\right)$ with $g(D)<1, g=1$ on $\pi(F)$, and $-1<g \leqq 1$. If $f \in F_{T}$ and $\rho(f)=g$, then $f(x)<1, f=1$ on $F$, and $-1<f \leqq 1$.

\section{REFERENCES}

[A] R. Atalla, On the multiplicative behavior of regular matrices, Proc. Amer. Math. Soc. 26 (1970), 437-446. MR 42 \#6633. 
1973] MARKOV OPERATORS, PEAK POINTS, AND CHOQUET POINTS 109

[L] G. Leibowitz, Lectures on complex function algebras, Scott, Foresman, Glenview, IIl., 1970.

[LI] S. P. Lloyd, On certain projections in spaces of continuous functions, Pacific J. Math. 13 (1963), 171-175. MR 27 \#2845.

[P] R. R. Phelps, Lectures on Choquet's theorem, Van Nostrand, Princeton, N.J., 1966. MR 33 \#1690.

[Sem] Z. Semadeni, Banach spaces of continuous functions, Vol. I, PWN, Warsaw, 1971.

[S] R. Sine, Geometric theory of a single Markov operator, Pacific J. Math. 27 (1968), 155-166. MR 39 \#1630.

Department of Mathematics, Ohio University, Athens, Ohio 45701 